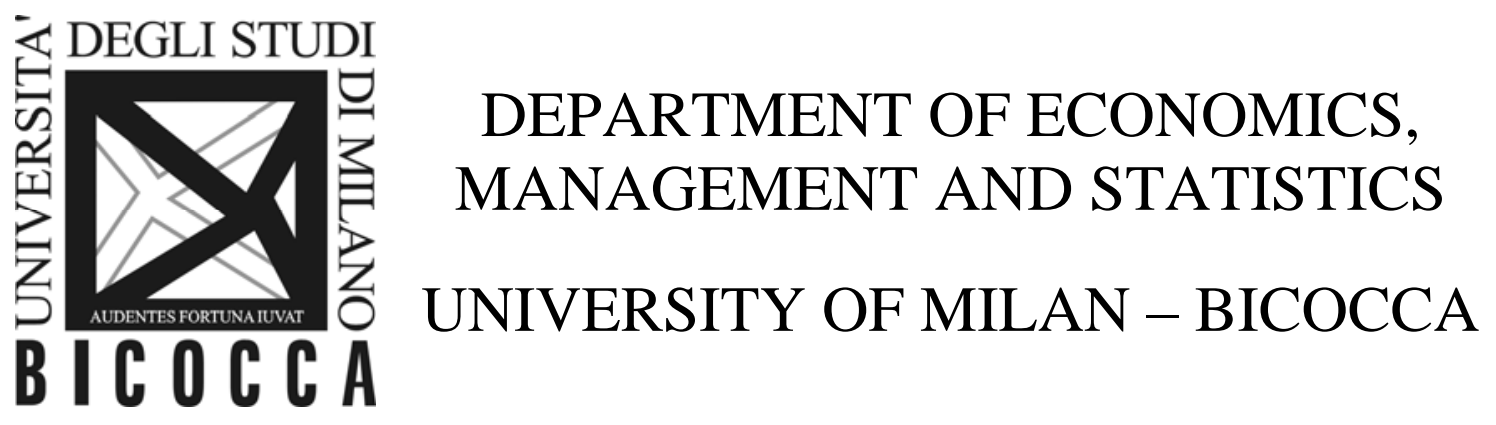

DEMS WORKING PAPER SERIES

A model of monopoly with lags in the planning and production activity

Fausto Cavalli

No. 326 - February 2016

Dipartimento di Economia, Metodi Quantitativi e Strategie di Impresa Università degli Studi di Milano - Bicocca

http://dems.unimib.it/ 


\title{
A model of monopoly with lags in the planning and production activity
}

\author{
Fausto Cavalli*
}

\begin{abstract}
In this note, departing from the traditional static and fully rational economic agent setting, I study a dynamic model of a boundedly rational monopolist who, in a partially known environment, follows a rule of thumb learning process. Instead of considering the classical differential model with smooth argument, the proposed dynamic model consists of a piecewise constant argument differential equation, in order to take into account the more realistic assumption of a lag between the learning activity and the output production activity. It is shown how this simple first order differential equation can be rephrased into a nonlinear difference equation which, differently from the classical model with smooth argument, can exhibit complex behaviors. The aim of the paper is to illustrate, from a methodological point of view, the potential applications and the dynamical effects of piecewise constant argument differential equations in economics.
\end{abstract}

Keywords: Monopoly, lags, bounded rationality, piecewise constant argument differential equation, complex dynamics.

JEL codes: D42, L12, C02, C62, C63, C65

\section{Introduction}

The traditional economic agent model is based on the assumption of rationality, that is, agents make their choices on the basis of the optimization of an objective function, subject to budget constraints. This approach requires that agents have a complete knowledge of the objective function and have the computational skills to solve the optimization problem. Even if a monopolistic market is a quite simple economic framework, this assumption would at least require the global knowledge of the market demand function. As several authors underlined, "ignorance about demand conditions is a ubiquitous feature of market life in the real world", so that the assumption of "ignorant monopolists" seems to be more realistic (Clower [1]). Simple learning mechanisms have been proposed, in

*Address: Department of Economics, Management and Statistics, University of MilanoBicocca, Piazza dell'Ateneo Nuovo 1, 20126, Milano, Italy, e-mail: fausto.cavalli@unimib.it 
which the firm, endowed with reduced rationality, tries to modify output levels toward the profit maximizing output, following rule-of-thumb mechanisms (see for example $[1,2])$. This means that the profit-maximizing equilibrium is not reached in one shot, but on the basis of repeated choices following a dynamic adaptive process (see Colisnk [3]), which requires minimal informational endowment and computational skills. In fact, in this case only a local knowledge of the demand function is required, which can be for instance obtained through periodical market experiments and surveys. In the long run, the convergence of such adaptive process toward the equilibrium point of the traditional static consumer model may be seen as an "evolutionary explanation" of the assumption of rational behavior.

Similarly to what done in $[4,5,6,7,8,9]$, in this paper a simple dynamic model of a monopolistic firm is studied. Time by time, the firm updates output decisions in the direction of increasing profit. However, it is more realistic to assume that the learning activity of data collection and organization, in order to have a formal representation of the profitability situation, only periodically occurs, at a different time scale with respect to that of the production decisional mechanism. Then, it is assumed that the learning process introduces lags in the planning activity. To this end, the learning activity is assumed to take place only at integer period times $t$, and it remains the basis of output decisions for the whole interval $[t, t+1)$. From the analytical viewpoint, this can be suitably represented through piecewise constant argument differential equations.

Differential equations with piecewise constant arguments (DEPCA) are used to model several real world phenomena. Since the early 1980's such dynamical systems have been applied in scientific fields such as engineering, physics, chemistry and biomedicine. The first model based on DEPCA was proposed in the biomedicine context by Busenberg and Cooke (1982) in [10]. Subsequently, the study of DEPCA and their applications have been considered in other fields: for a survey, we refer to [11] and to the references therein. The most interesting aspect about solutions of such systems is that they combine the features of both differential and difference equations.

The model proposed in this paper represents, to the best of knowledge, a first attempt toward the application of piecewise constant argument differential equations to an economic context. The resulting dynamical model generalizes the underlying static model, because the profit-maximizing equilibrium belongs to the set of the steady states of the dynamic system. The most significant aspect of the proposed approach is that, differently from the classical differential model in which no lags in the planning activity are introduced, the dynamic modeling based on DEPCA introduces a rich variety of behaviors which can lead, through a sequence of period-doubling bifurcations, to complex dynamics. This is essentially due to the peculiar time characterization of the model, in which two different time scales, a continuous and a discrete one, are involved. The resulting model can be seen as a bridge model between classical continuous and discrete time models.

The remainder of the paper is organized as follows: in Section 2 the canonical static monopoly model is presented, in Section 3 the dynamic monopoly model 
with learning is considered and in Section 4 some possible further applications of the proposed approach are illustrated.

\section{The Monopoly model}

The relevant feature of a monopoly model is that a single seller, the monopolist, faces price-taking consumers in a market over one or several periods. This means that the monopolist, who has market power, can consequently either determine the price for the product or the supplied quantity (output level). Since price and output are related through demand function, a decision about price implies a decision about quantity and vice-versa. In what follows, I shall focus on the case of a monopolist that, in order to maximize his profit, sets the output level, described by variable $q>0$.

The monopolist, in order to choose his price and output level, faces two kinds of constraints. Firstly, he faces an internal constraint, imposed by his technological structure, which can be analytically represented by the cost function $c(q)$, which relates the quantity produced to the cost of producing that quantity. Secondly, he faces an external constraint, represented by the consumer's behavior, encompassed into the demand function, which relates the market price to the quantity produced and supplied by the monopolist. As usual, the natural way to set the monopolist's optimization problem is to consider the inverse demand function $p(q)$, which defines the price that must be charged to sell $q$ units of output, and to look for the optimum output level which maximizes the profit function

$$
\pi(q)=p(q) q-c(q) .
$$

In what follows, a linear demand function is considered

$$
p(q)=a-b q, a>0, b>0,
$$

together with a quadratic, increasing cost function

$$
c(q)=d q+e q^{2},
$$

in which parameters $d$ and $e$ have to satisfy $d+2 e q>0$ for all the feasible output levels $q>0$. Profit function

$$
\pi(q)=(a-d) q-(b+e) q^{2}
$$

is then maximized by a strictly positive output level $q^{*}$ provided that first order condition $a-d-2(b+e) q^{*}=0$ is satisfied, being second order condition $2(b+e)>0$ always fulfilled. Assuming that $a>d$, the maximizing output level and the corresponding optimal price are then the strictly positive quantities

$$
q^{*}=\frac{a-d}{2(b+e)}, p^{*}=a-b\left(\frac{a-d}{2(b+e)}\right) .
$$

It must, only the case of internal optimal output level is considered. The remaining situation in which $q^{*}=0$ could be considered as well, but since it is economically less interesting, it will not be investigated further. 


\section{Monopoly model with learning}

The assumption of optimizing behavior requires that economic agents have high computational capabilities and a set of complete information about the environment. Actually, the monopolist has a limited and local knowledge of the demand function. The lack of information is due to the costly and time consuming nature of the collecting information activity. It is then more realistic to assume that the monopolist is not able to reach the equilibrium in one shot and, consequently, acts on the basis of a local estimate of the marginal profit $\pi^{\prime}$, obtained, for example, through market experiments. The adaptive process consists of a learning activity and of revising decisions after considering past output levels and outcomes. One can suppose that the monopolist employs a rule of thumb as a local (or myopic) profit maximizer. In particular, he looks at how a variation in the supplied quantity affects the variation of profits. A positive (negative) variation of profits will induce the monopolist to change the quantity in the same (opposite) direction from that of the preceding period. If profits are stationary, the output level is not changed. In this setting, the time evolution is continuous and the firm takes output decisions continuously. Conversely, the firm carries out a periodical learning activity of collection and organization of information and data about the environment in which it acts, in order to produce a formal representation of the profitability situation (marginal profit). However, it is not realistic to assume that the learning activity takes place continuously, with the same timing of the production activity. On the contrary, it can be supposed that it only periodically takes place, for example at discrete times $n=0,1,2, \ldots$, so that output decisions, during each time period $n \leq t<n+1, n=0,1,2 \ldots$, are based on the representation given by the learning activity carried out at time $t=n$. In other words, the decisional process has two different temporal: the former in which output decisions are taken and updated and which is suitably described in terms of a continuous time evolution, and the latter in which learning activity takes place and which is better modeled through a discrete time evolution. The whole decisional mechanism can be represented through the following nonlinear equation with piecewise constant argument

$$
\frac{d q(t)}{d t}=k(q(t)) \frac{d \pi}{d q}[t], t \geq 0, q(0)=q_{0}>0,
$$

where $[t]$ denotes the largest previous integer with respect to $t$ and $k(q)$ is a positive and increasing function which describes the extent of production variation of the monopolist following a certain profit signal and depends on the current monopolistic firm dimension, given by the production volume. In what follows, the simple linear form

$$
k(q(t))=v q(t)
$$

is assumed, where $v$ is a positive parameter which gives the relative speed of adjustment. Considering linear demand function (1) and quadratic cost function 
(2), equation (4) becomes

$$
\frac{d q(t)}{d t}=v q(t)[a-d-2(b+e) q[t]], t \geq 0, q(0)>0 .
$$

Due to the presence of the discontinuous term $[t]$ in the right hand side of equation (4), a particular definition of solution of (4) is required. In particular, a solution of a DEPCA, as precised in [12], has to be a function $q:[0,+\infty) \rightarrow$ $(0,+\infty)$ that for each $n \in \mathbb{N}$

(A) is continuous on $[0,+\infty)$;

(B) is differentiable on each interval $(n, n+1)$, with one-sided derivatives that exist for $t=n$;

(C) solves the smooth argument differential equation (4) on each $[n, n+1)$.

The analytical solution of problem (6) is studied in the following proposition.

Proposition 1. Let $q(0)=q_{0}>0$. Then there exists a unique solution of problem (6) given by

$$
q(t)=q(n) \exp [v(a-d-2(b+e) q(n))(t-n)], t \in[n, n+1), n \in \mathbb{N} .
$$

Proof. As shown in Theorem 2.1 in [12, Ch. 2], a DEPCA of the form $x^{\prime}(t)=$ $f(x(t), x[t]), x(0)=x_{0}$, has a unique solution on $[0,+\infty)$ provided that, for each $\mu \in \mathbb{R}$, the solution of the classical differential equation $x^{\prime}(t)=f(x(t), \mu)$ exists and is unique on the whole interval $[0,+\infty)$. Concerning $(6)$, this requires considering the linear Cauchy problem

$$
x^{\prime}(t)=v x(t)(a-d-2(b+e) \mu), x(0)=q_{0},
$$

which indeed has a unique global solution. Then, there exists a unique solution of DEPCA (6). It is easy to show that (7) satisfies assumptions (A) and (B) and solves equation (4).

Thanks to the continuity of the solution of (4), one can write

$$
\begin{aligned}
q(n+1) & =\lim _{t \rightarrow(n+1)^{-}} q(t) \\
& =\lim _{t \rightarrow(n+1)^{-}} q(n) \exp [v(a-d-2(b+e) q(n))(t-n)] \\
& =q(n) \exp [v(a-d-2(b+e) q(n))],
\end{aligned}
$$

which allows associating, with the continuous time differential problem (4), a discrete difference equation, which describes the evolution of $q(t)$ at discrete times. Setting $q(n)=q_{n}$ for $n \in \mathbb{N}$, from (8) one can obtain the discrete difference equation

$$
q_{n+1}=f\left(q_{n}\right)=q_{n} \exp \left[v\left(a-d-2(b+e) q_{n}\right)\right], n \geq 0 .
$$




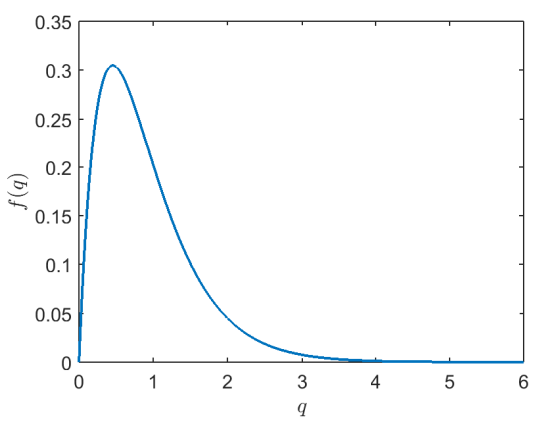

Figure 1: Plot of $f(q)$.

It is interesting to notice that the original linear, additive, boundedly rational continuous time mechanism described by (6) has now become a nonlinear, multiplicative, boundedly rational discrete time mechanism. However, both mechanisms (6) and (8) represent a rule of thumb. In fact, from (4), $q(t)$ increases (resp. decreases) as $\pi^{\prime}(q[t])=a-d-2(b+e) q[t]$ is positive (resp. negative), as well as from (9), $q_{n+1}$ increases (resp. decreases) as $\exp \left(a-d-2(b+e) q_{n}\right)>1$ (resp. $\left.\exp \left(a-d-2(b+e) q_{n}\right)<1\right)$, or, equivalently, again as $\pi^{\prime}\left(q_{n}\right)>0$ (resp. $\pi^{\prime}\left(q_{n}\right)<0$ ). It's worth noticing that $f(q)$ is a positive function, vanishing for $q \rightarrow+\infty$, increasing for $q \in(0,1 /(2 b v+2 e v))$ and decreasing for $q \in(1 /(2 b v+2 e v),+\infty)$ (see Figure 1 for an example of plot, obtained setting $a=1, b=0.1, d=0.4, e=1$ and $v=1)$. The properties of (9) are summarized in the following proposition.

Proposition 2. Let $q_{0}>0$. The only steady state of (9) is the optimal output level $q^{*}$ given by (3), which is locally asymptotically stable provided that

$$
v(a-d)<2 .
$$

Proof. Steady states are obtained by setting $f(q)=q$, from which one obtains $q=0$, which is excluded since $q_{0}>0$, and $\exp (v(a-d-2(b+e) q))=1$, which provides $q=q^{*}$. Local asymptotic stability of a steady state $x$ is obtained imposing $\left|f^{\prime}(x)\right|<1$. Since

$$
f^{\prime}\left(q^{*}\right)=1-v(a-d),
$$

the proof is complete.

Condition (10) simply says that the steady state can be unstable if either reaction speed $v$ or $a-d$ are sufficiently large. Instable dynamics can then occur if a too reactive behavior is adopted by economic agents, as well as if, for example, the market size (influenced by $a$ ) is large. A simple example of unstable dynamics is shown in the bifurcation diagrams with respect to $v$ and $a$ reported in Figure 2, obtained considering $b=0.1, d=0.4, e=1$, as in Figure 1. 


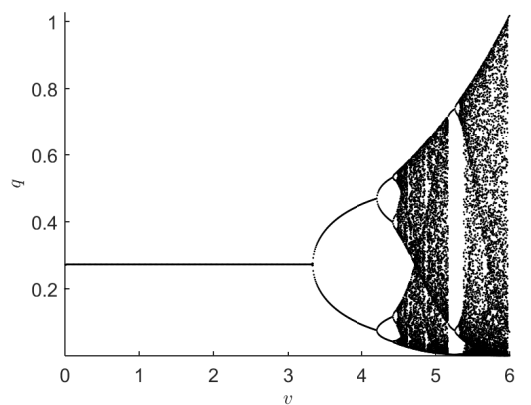

(a)

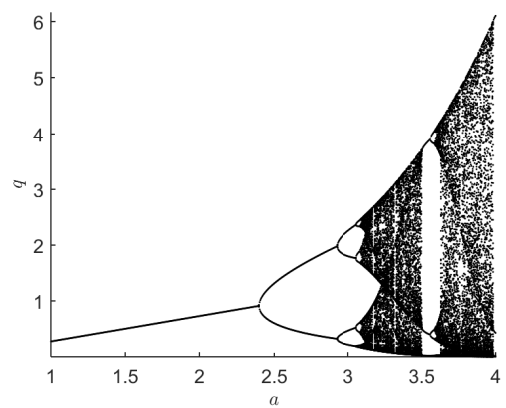

(b)

Figure 2: (a): Bifurcation diagram for (9) on varying $v$. (b): Bifurcation diagram for (9) on varying $a$.

Using the new state variable $x=q /(2(b+e))$, equation (9) can be rewritten as

$$
x_{n+1}=x_{n} \exp \left[\beta-x_{n}\right],
$$

where $\beta=a-d$. Equation (11) is a well-known first order nonlinear difference equation, with a rich variety of dynamical behaviors, often used in biology to predict the size of a population after $t$ discrete time steps, and has been proposed by Ricker in [13] (see also [14, 15, 16, 17, 18, 19]). In ecological modeling, the state variable $x_{t}$ denotes population size (density) at time $t$ and the parameter $\beta>0$ describes the intrinsic growth rate. There is a wide analytical literature about the dynamical behavior of Ricker's map [20, 21]. Summarizing, Ricker's map has a fixed point $x^{*}=\beta$, that is globally attracting provided that $0<$ $\beta<\beta_{1}=2$. As $\beta$ increases the fixed point can lose stability and a cascade of period-doublings occurs. When $\beta \approx \beta_{2}=2.5264$, an attracting period-2 cycle appears. It is possible to show that the sequence of period doubling bifurcations occurs for parameter values forming a sequence $\beta_{n}$ with $\lim _{n \rightarrow+\infty} \beta_{n}=\beta_{+\infty}$, where $\beta_{+\infty}=2.6294$. For more details, see $[20,21]$ and the references therein.

\section{Conclusions}

In this note a simple dynamic model of a monopolistic firm is presented, in which the decisional mechanism is represented by a gradient-like rule of thumb. It is assumed that at each time the firm updates output decisions in the direction of increasing profits and that there is, in all integer time periods, a learning activity that represents the basis of output decisions until the next integer time period. Such a formal representation of the decisional processes is modeled in a natural way through a piecewise constant argument differential equation. Of course, there are other areas of economic analysis that can benefit from the piecewise constant argument equation approach. An economic agent, firm or 
consumer, before taking a decision has to produce, periodically, a formal representation of the environment in which he acts; such learning activity introduces a dependence between the variation of the control state variable and data collected in a previous time period. From this point of view it will be interesting to use this approach in game theory when modeling players involved in a strategic interaction with other players. In such case, the learning activity can concern not only prices and economic quantities, but also the other players' choices.

\section{References}

[1] R. W. Clower. Some theory of an ignorant monopolist. Economic Journal, 69(276):pp. 705-716, 1959.

[2] W. J. Baumol and R. E. Quandt. Rules of thumb and optimally imperfect decisions. American Economic Review, 54(2):23-46, 1964.

[3] J. Colinsk. Why bounded rationality. Journal of Economic Literature, 34:669-700, 1996.

[4] T. Puu. The chaotic monopolist. Chaos, Solitons Fractals, 5(1):35-44, 1995.

[5] A. Naimzada and G. Ricchiuti. Complex dynamics in a monopoly with a rule of thumb. Applied Mathematics and Computation, 203(2):921-925, 2008.

[6] A. Matsumoto and F. Szidarovszky. Nonlinear delay monopoly with bounded rationality. Chaos, Solitons Fractals, 45(4):507 - 519, 2012.

[7] S. S. Askar. On complex dynamics of monopoly market. Economic Modelling, 31(1):586-589, 2013.

[8] A. Matsumoto and F. Szidarovszky. Complex dynamics of monopolies with gradient adjustment. Economic Modelling, 42:220-229, 2014.

[9] B. Al-Hdaibat, W. Govaerts, and N. Neirynck. On periodic and chaotic behavior in a two-dimensional monopoly model. Chaos, Solitons Fractals, $70: 27-37,2015$.

[10] K. Busenberg and L. Cooke. Models of vertically transmitted diseases with sequential-continuous dynamics. In V. Lakshmikantham, editor, Nonlinear Phenomena in Mathematical Sciences, pages 179-187. Academic Press, New York, 1982.

[11] K. L. Cooke and J. Wiener. A survey of differential equations with piecewise continuous arguments. In Delay Differential Equations and Dynamical Systems. Springer, 1991. 
[12] J. Wiener. Generalized Solutions of Functional Differential Equations. World Scientific, 1993.

[13] W. E. Ricker. Stock and recruitment. Journal of the Fisheries Research Board of Canada, 11:559-623, 1954.

[14] B. S. Fisher, M. E. Goh and T. L. Vincent. Some stability conditions for discrete-time single species models. Bulletin of Mathematical Biology, 41:861-875, 1979.

[15] B. S. Goh. Stability in a stock recruitment model of an exploited fishery. Mathematical Biosciences, 33:359-372, 1977.

[16] R. N. Greenwell and H. K. Ng. The Ricker salmon model. The UMAP Journal, 5:339-359, 1984.

[17] A. Hastings. Population Biology: Concepts and Models. Springer-Verlag New York, Inc., 1997.

[18] R. M. May. Biological populations with non-overlapping generations: Stable points, stable cycles and chaos. Science, 186:645-647, 1974.

[19] R. M. May and G. F. Oster. Bifurcations and dynamic complexity in simple biological models. The American Naturalist, 110:573-599, 1976.

[20] S. Elaydi and R. Sacker. Basin of attraction of periodic orbits of maps on the real line. Journal of Difference Equations and Applications, 10:881-888, 2004 .

[21] P. Liu and K. Gopalsamy. Global stability and chaos in a population model with piecewise constant arguments. Applied Mathematics and Computation, 101:63-88, 1999. 